\title{
Publisher Correction: Cluster richness-mass calibration with cosmic microwave background lensing
}

James E. Geach and John A. Peacock

Correction to: Nature Astronomy https://doi.org/10.1038/s41550-017-0259-1 (2017); published online 9 October 2017.

Owing to a technical error, the 'Additional information' section of the originally published PDF version of this Letter incorrectly gave J.A.P. as the corresponding author; it should have read J.E.G. This has now been corrected. The HTML version is correct.

Published online: 31 January 2018

https://doi.org/10.1038/s41550-017-0354-3 